University of Wollongong

Research Online

Australian Institute for Innovative Materials -

Papers

Australian Institute for Innovative Materials

$1-1-2017$

System and process development for coaxial extrusion in fused deposition modelling

Adam Taylor

University of Wollongong, taylora@uow.edu.au

Stephen T. Beirne

University of Wollongong, sbeirne@uow.edu.au

Gursel Alici

University of Wollongong, gursel@uow.edu.au

Gordon G. Wallace

University of Wollongong, gwallace@uow.edu.au

Follow this and additional works at: https://ro.uow.edu.au/aiimpapers

Part of the Engineering Commons, and the Physical Sciences and Mathematics Commons

Research Online is the open access institutional repository for the University of Wollongong. For further information contact the UOW Library: research-pubs@uow.edu.au 


\title{
System and process development for coaxial extrusion in fused deposition modelling
}

\author{
Abstract \\ Purpose: This paper aims to design and test a system capable of coaxial fused deposition modelling \\ (FDM) and assess the coaxial fibres produced for their coaxial concentricity. The goal is to achieve \\ concentricity values below the literature standard of 15 per cent. Design/methodology/approach: This \\ research discusses the design of the coaxial nozzle internal geometry and validates the modelling \\ process by using computational fluid dynamics to assess its flow profile. Sequentially, this paper \\ discusses the abilities of current additive manufacturing (AM) technology in the production of the coaxial \\ nozzle. Findings: The methodology followed has produced coaxial fibres with concentricity values as low \\ as 2.89 per cent and also identifies a clear speed suitable for coaxial printing using polylactic acid (PLA) \\ as the internal and external materials. Research limitations/implications: The concentricity of the printed \\ fibres is heavily influenced by the feed rate for the thermoplastic feedstock. This in turn alters the \\ viscosity of the material to be printed, implying that a relationship exists between feed rate and print \\ temperature, which can be further optimised to potentially obtain higher concentricity values. Practical \\ implications: This paper adds reliability and repeatability to the production of coaxially printed structures, \\ the likes of which has numerous potential applications for biological printing. Originality/value: The \\ outcomes of this study will provide an AM platform to alter the paradigm of biofabrication by introducing \\ a new level of versatility to the construction of biofabricated structures.

\section{Disciplines} \\ Engineering | Physical Sciences and Mathematics

\section{Publication Details} \\ Taylor, A. C., Beirne, S., Alici, G. \& Wallace, G. G. (2017). System and process development for coaxial \\ extrusion in fused deposition modelling. Rapid Prototyping Journal, 23 (3), 543-550.
}




\title{
System and Process Development for Coaxial Extrusion in Fused Deposition Modelling
}

\begin{abstract}
Purpose - This paper aims to design and test a system capable of coaxial fused deposition modelling (FDM) and assess the coaxial fibres produced for their coaxial concentricity. The goal is to achieve concentricity values below the literature standard of $15 \%$.

Design/methodology/approach - This research discusses the design of the coaxial nozzle internal geometry and validates the modelling process by using computational fluid dynamics to assess its flow profile. Sequentially, this paper discusses the abilities of current additive manufacturing technology in the production of the coaxial nozzle.

Findings - The methodology followed has produced coaxial fibres with concentricity values as low as $2.89 \%$ and also identifies a clear speed suitable for coaxial printing using poly lactic acid as the internal and external materials.

Research limitations/implications - The concentricity of the printed fibres is heavily influenced by the feed rate for the thermoplastic feedstock. This in turn alters the viscosity of the material to be printed, implying that a relationship exists between feed rate and print temperature which can be further optimised to potentially obtain higher concentricity values.

Practical implications - This paper adds reliability and repeatability to the production of coaxially printed structures, the likes of which has numerous potential applications for biological printing.

Originality / value - The outcomes of this study will provide an opportunity to alter the paradigm of biofabrication by introducing a new level of versatility to the construction of biofabricated structures.
\end{abstract}

Keywords: Coaxial fused deposition modelling, fused deposition modelling, FDM, Computation Fluid Dynamics, Design Optimisation, Coaxial

Paper Type: Research Paper

\section{INTRODUCTION}

\subsection{ADDITIVE MANUFACTURING}

Additive manufacturing (AM) is defined as a "process of joining materials to make objects from threedimensional model data, usually layer upon layer" [1]. This process, also known as three-dimensional printing or additive fabrication, is an emerging technology finding applications across a range of industries. When comparing this method with traditional manufacturing, it is evident that AM relies on the concept of addition of material where required, while traditional methods remove unwanted material to create the desired geometry. This results in a process that is more efficient from a material consumption perspective, though it has shown to lack efficiency in time to manufacture [2].

AM services a variety of industries including, aerospace [3, 4], automotive [5], biomedical [6-8] and tooling [9]. Each sector utilizes the desired method for a number of reasons; though a common theme is the ability to manufacture using materials with unique properties [10-14]. AM processes can use materials 
which are biocompatible, biodegradable, conductive or insulating; while varying the density of the materials deposition. However, despite the recent expansion of research and development into these techniques, additional progression needs to be made to further enhance their capabilities.

\subsection{COAXIAL ADDITIVE MANUFACTURING}

A coaxial structure is one in which two or more materials are present and share a common axis. This structural configuration is commonly found in conducting cables, where the internal material is conductive in nature and the external is insulating, protecting the core from the environment and permitting the desired transfer of the signal. In the scenario of FDM, coaxial fibres will take the form of concentric circles, retaining the fibre and sheath configuration.

Individual and sequentially controlled co-extrusion is a common feature for new FDM devices [15], typically allowing for the deposition of a model material from one print tip and a support material from another. While this method is of great benefit for producing easy to remove support, or multi-material structures, the concept fails to compete with the complexity and versatility which could be achieved through a coaxial deposited fibre. Additionally, the strength characteristics of a multi-material printed structure would likely be lower than a single material build as the process will introduce a mechanical weakness at the point at which the two materials meet. A coaxial fibre, however, would allow the two separate materials to fuse while still in a molten state, altering the binding process between them and likely their strength characteristics.

The first research into coaxial extrusion techniques in AM was completed in 2010 [16]. This work developed a means to produce a three-dimensional porous collagen scaffold with an alginate core. The scaffold was created through a solid freeform fabrication of collagen and alginate coupled with a cryogenic and freeze-drying system. The coaxial nozzle had an inner diameter of $175 \mu \mathrm{m}$ and an outer diameter of $500 \mu \mathrm{m}$ with the resultant structures showing a high level of dimensional accuracy. However, no measurements were made regarding the concentricity of the coaxial fibres, with a majority of the data suggesting the structures were not symmetrical about their longitudinal axis.

Subsequent work into coaxial extrusion in AM involved the modification of a bioprinting device, a similar concept to FDM though commonly used for low temperature biological fluids. This research primarily targeted coaxial extrusion of organic materials and their incorporation into scaffold structures; specifically, the use of poly- $\varepsilon$-caprolactone (PCL) and a composite PCL graphene material [17]. The designed tip exhibits diameters of $900 \mu \mathrm{m}$ and $300 \mu \mathrm{m}$ for external and internal, respectively, though the fibres produced by this tip have dimensions of $800-700 \mu \mathrm{m}$ and $460-300 \mu \mathrm{m}$ for external and internal materials, respectively.

The previous work considers the importance of developing an accurate, reliable and repeatable process in producing coaxial fibres through an AM process. The reliability of the device is assessed by measuring the offset of the core materials longitudinal axis from the external material's longitudinal axis. The bioprinting coaxial process reports axis offset averages as low as $15 \%$, though the method for obtaining this percentage is not defined.

The concept of coaxial extrusion has had limited development due to the complex geometry required for the coaxial hot-end. The resolution required and structural features would be impossible to construct using traditional manufacturing methods. Due to advances in additive manufacturing, it is now possible to produce such a component in metal (a titanium alloy (Ti6Al4V) will be used for ease of fabrication) through selective laser melting (SLM). This metal printing process acts as the catalyst for the design and construction of this customised printer. 


\section{EXPERIMENTAL DESIGN}

\subsection{Extruder Design}

When designing the new extrusion mechanism, a key constraint was imposed regarding the angular orientation of the coaxial nozzle. As this intricate and complex component was to be fabricated by selective laser melting, the angular constraint was linked to the system's ability to print angled structures without requiring a support material. It is crucial to obtain optimum flow within the nozzle design; this demands that no internal support material be present within the coaxial nozzle chambers. This ultimately fixed the feed mechanism's position at an orientation of $45^{\circ} \leq$ from the vertical, limiting the potential configurations for the extruder position as new spatial constraints are now imposed.

Figure 1a) shows the geared extrusion design concept. This system works by applying a force (F) to a bearing housing (A) which causes a feedstock (B) to make contact with a toothed gear (C). The toothed gear then grips the feedstock and propels it in the desired direction. The bearing acts to reduce undesirable friction, while the contact force, $\mathrm{F}$, is adjustable as a result of a spring mechanism shown in Figure 1b).

An additional constraint of the extruder design is the requirement to supply feedstock for two materials simultaneously. Given the nature of the coaxial nozzle design, which is defined in the following section, the two materials must be supplied at differing rates. This implies that two extrusion systems are required and there must be independent control over the speed of each, eliminating the possibility of utilising a single stepper motor to supply both feedstock materials.
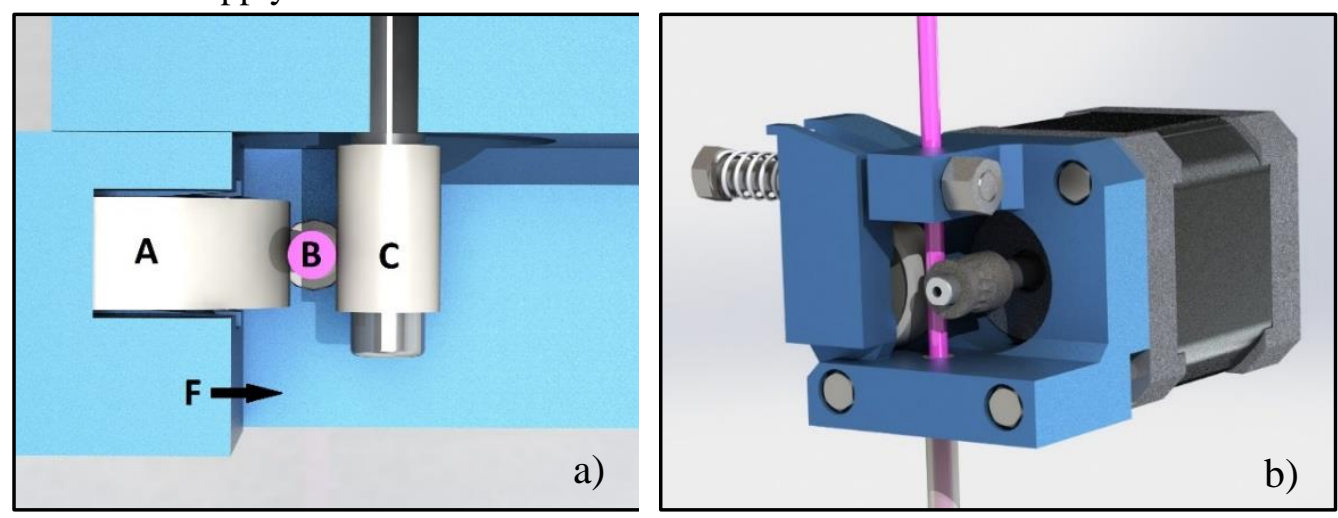

Figure 1 - CAD models of designed extruder.

a) Top-down view: Improved geared extruder design. b) Feed mechanism

\subsection{Design Optimization of Coaxial Nozzle}

To create a system which produces coaxial flow, the external material must envelope the passage of the internal material before allowing the two fluids to interface. Previous work into coaxial extrusion produced coaxial fibres with minimum offsets of $15 \%$. This paper explores the impact of accurately predicting fluid flow with the expectation that this will optimize the concentricity of the printed materials; a key goal for this work. This section focuses on the design constraints imposed on producing a coaxial hot-end and the process of creating its internal geometrical curvature. To optimize the flow performance of the coaxial nozzle design, several nozzles were created using Solidworks to generate three-dimensional models, and ANSYS CFX used to compute thermal and flow simulations on the concept designs.

The model geometries varied in the external fluid pathway only and were designed systematically as governed by the following conditions:

1. Straight pathway for external fluid (Figure 2a)) 


\section{System and Process Development for Coaxial Extrusion in Fused Deposition Modelling}

2. Create $5^{\circ}$ angular cross-sections which are perpendicular to the external fluid pathway and span a total of $50^{\circ}$. Progressively reduce the cross-sectional area of the external fluid pathway by an equal amount until the internal fluid pathway is concentric within the external pathway.

3. Following the external fluid pathway and using the $5^{\circ}$ method from condition $\# 2$, progressively expand the cross-sectional area in an elliptical form until the internal fluid pathway has been enveloped by the external, but is not yet concentric.

4. Identical methodology as condition \#3 though the cross-sections were based on the internal fluid pathway rather than the external (Figure 2b)).

The initial CAD model (Figure 2a)) was created for the coaxial nozzle as a bench mark, it exhibited no geometrical variation and its flow characteristics were simulated. The result (Figure 2c)) identified an imbalanced velocity distribution and a region of static flow. This places a strong emphasis on equalizing the velocity distribution as the two fluid channels become concentric and minimizing static flow where possible. While it is likely that these flow conditions will change depending on the material being processed, for the nature of this work, the materials used in simulations will be PLA or ABS as these are the most common thermoplastics used in FDM systems and will allow for higher temperature testing.
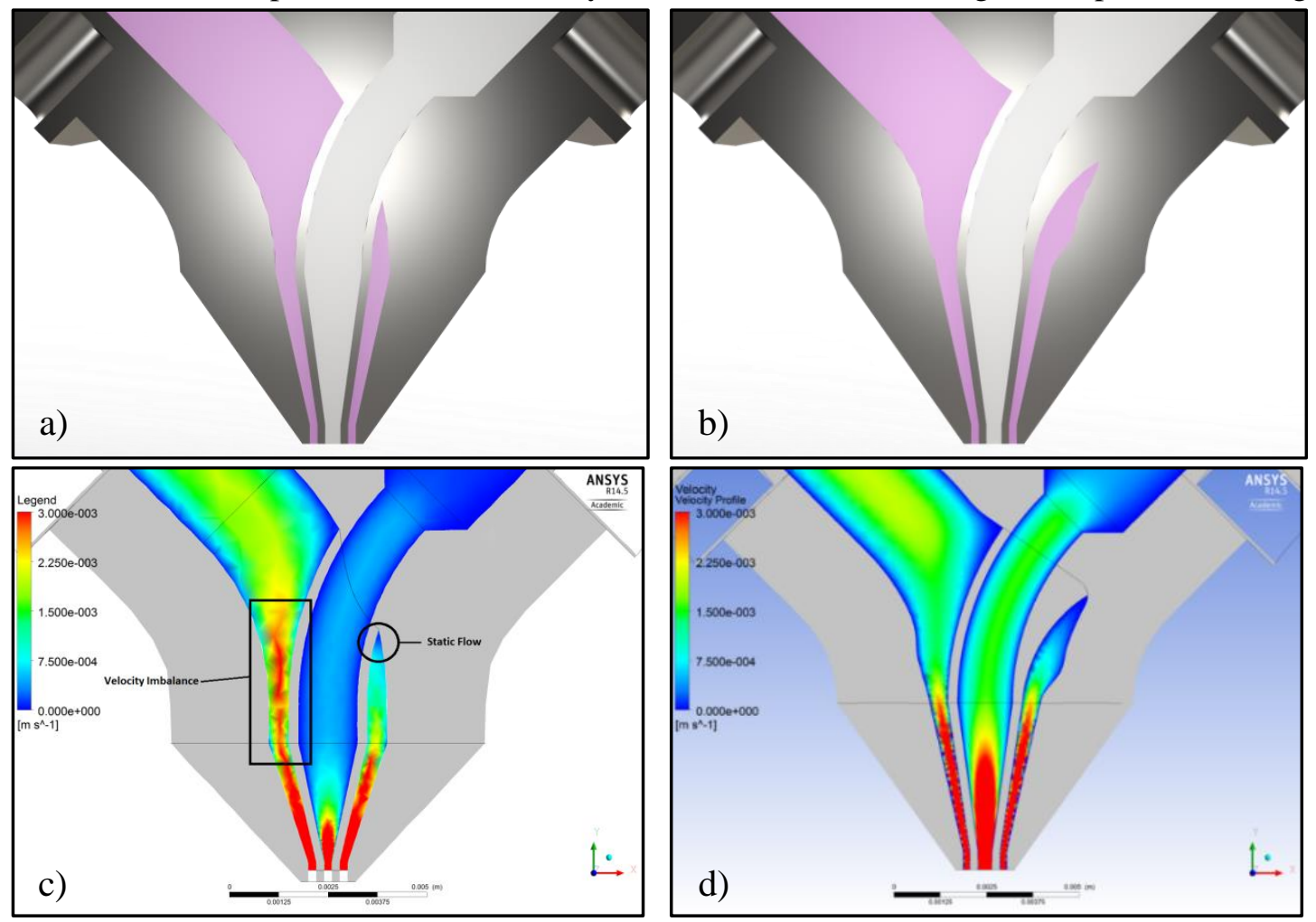

Figure 2 - Modelled fluid pathways and the associated fluid velocity profiles.

a) Straight pathway for external fluid. b) Geometry based on condition \#4. c) CFD simulations conducted on a) using internal material as ABS, external material as PLA identify regions of static flow and velocity imbalance. d) The impact of an aerofoil design, and geometrical curvature based on b), on velocity imbalance and static flow

Depending on the viscosity of the material to be printed and the speeds at which they will be processed, areas of static flow occur to some extent in all geometries similar to that shown in Figure 2c). This region is found as the internal nozzle enters into the external chamber; the protruding structure obstructs flow and creates a surface shielded from the direction of fluid flow. As a result, a solid aerofoil shape has been modelled around the internal nozzle in an attempt to minimize this occurrence and create a more uniform 
pressure distribution. The variation in static flow between a nozzle modelled on condition \#1 and one modelled on condition \#4 with an aerofoil is shown in Figure 2c) and d), respectively.

While the simulations aim to produce a more uniform pressure distribution and velocity, the ultimate goal is to achieve concentricity in the final print material. This is expected to be obtained by producing equal velocities for both the internal and external chambers. However, the merging of the materials outside of the nozzle may cause irregularities to appear in the print materials.

The simulations have been completed under the following circumstances. Let the material feedstock be either ABS or PLA, and the body of the coaxial hot-end be constructed from Ti6Al4V, as this is the metal powder which the coaxial tip will be fabricated from. The molar mass, density, specific heat capacity, dynamic viscosity and thermal conductivity for each material can be found in Table 1 . The heat chambers are set to either $468.15^{\circ} \mathrm{K}$ or $533.15^{\circ} \mathrm{K}$, as these are typical printing temperatures for PLA and ABS respectively. Outlet pressure is assumed to be $101,325 \mathrm{~Pa}$ at an ambient temperature of $298.15^{\circ} \mathrm{K}$. All simulations have been completed assuming laminar flow.Feedstock velocity for use in simulations has been determined from a baseline output velocity for the external material of $60(\mathrm{~mm} / \mathrm{min})$. The internal material feed rate has been calculated by obtaining a ratio of the tip output areas for internal and external pathways and applying the associated reduction to the external material feed rate.

Table 1 - CFX simulation parameters

\begin{tabular}{|c|c|c|c|}
\hline & ABS[18] & $\begin{array}{c}\text { PLA[19- } \\
\text { 21] }\end{array}$ & Ti6Al4V[22] \\
\hline Molar Mass (kg/mol)* & 0.211 & 0.0721 & 0.446 \\
\hline Density (kg/m ${ }^{3}$ ) & 1060 & 1251 & 4430 \\
\hline $\begin{array}{c}\text { Specific Heat Capacity } \\
\text { (J/Kg.K) }\end{array}$ & 1400 & 1800 & 0.5263 \\
\hline $\begin{array}{c}\text { Dynamic Viscosity } \\
\text { (Pa.s) }\end{array}$ & 5 & 5 & N/A \\
\hline $\begin{array}{c}\text { Thermal Conductivity } \\
\text { (W/m.K) }\end{array}$ & 0.23 & 0.11 & 6.7 \\
\hline
\end{tabular}

* As molar mass cannot be determined for ABS or PLA due to the unknown degree of polymerization of the materials skeletal structure, a single degree of polymerization has been selected to represent each material.

Using the defined parameters and testing the created models, prioritizing equal velocity profiles and minimizing regions of static flow, a final concept design was created as shown in Figure 2b). Figure 3a) shows the impact of localised heating and heat distribution about the flow profile for PLA in both the internal and external chambers. Figure 3b) shows the effect of a thermal gradient when printing materials with large temperature differences in melting temperature such as PLA and ABS; this simulation has been conducted as a worst-case-scenario for thermal gradients. Initial physical prints will use PLA for both internal and external materials for simplicity.

This indicates a fulfilled design criteria, showing an equal velocity profile for the external fluid about the central axis in Figure 3c). Concurrently, the simulation indicates that the aerofoil design for the protruding internal material minimizes the likelihood for the occurrence of regions of static flow. 


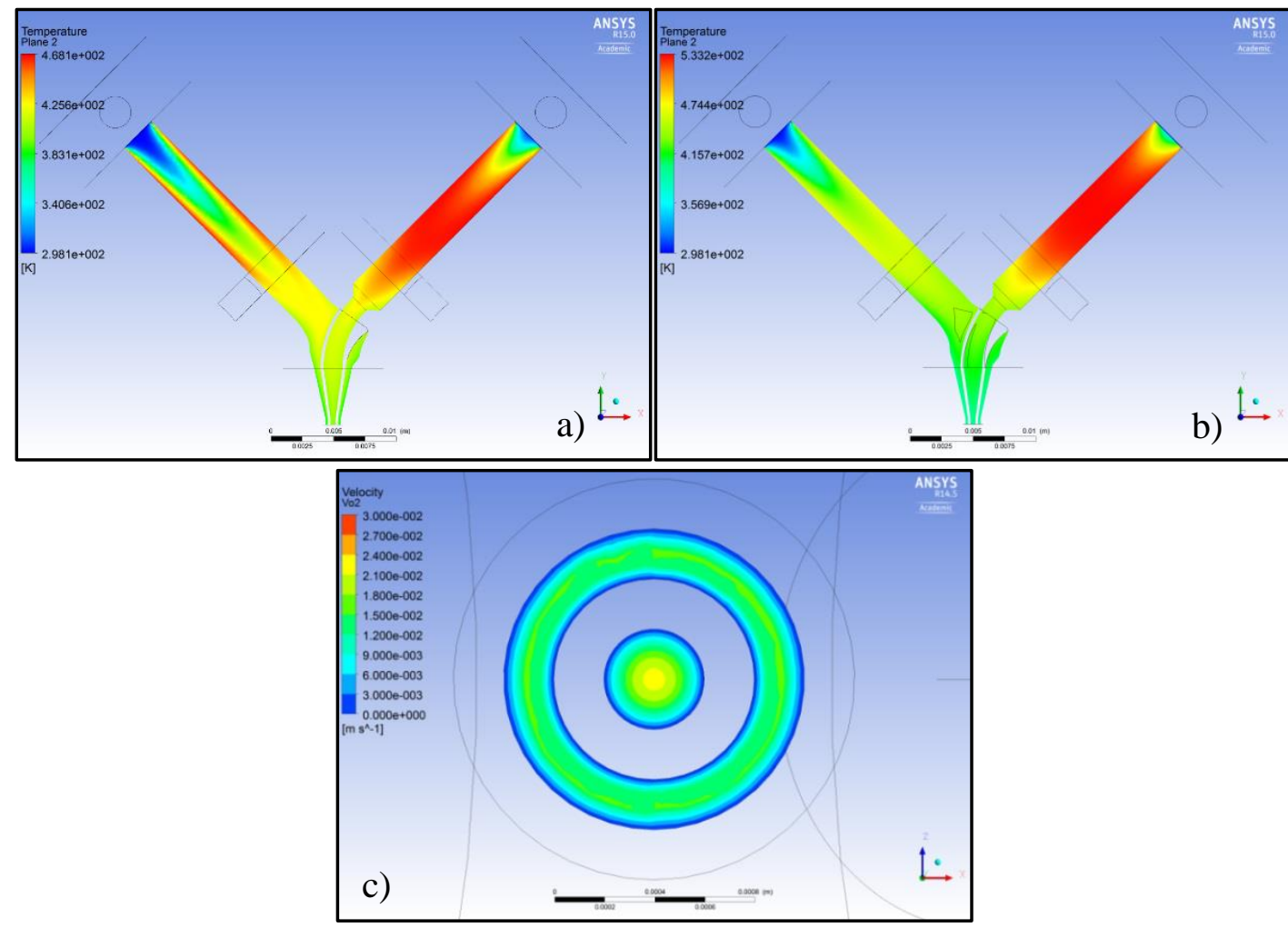

Figure 3 - Coaxial nozzle simulations,

a) Fluid thermal conductivity using PLA as both internal and external materials. b) Fluid thermal conductivity using ABS and PLA as the internal and external materials respectively. c) Bottom-up velocity profile

\section{Additive Manufacture Of Components}

\subsection{Fused Deposition Modelling - Base and Supporting Structures}

Additive manufacturing systems offer the ability to realize complex structures which would be otherwise impossible to manufacture using traditional methods. They offer rapid production with a variety of materials, making them an ideal solution for quick, complex designs. To take advantage of these abilities, a majority of the designed components were fabricated using the Australian National Fabrication Facilities (ANFF) owned UPrint Plus system.

This device enables the production of objects with a minimum layer thickness of $254 \mu \mathrm{m}$, while providing a variety of material fill density options. This infill density allows the printed structure to tolerate more force at the expense of increased object weight. All components manufactured through this method have been created with the minimum layer thickness at a high density infill using ABS as the build material.

\subsection{Selective Laser Melting - Coaxial Nozzle and the Dimensional Accuracy of the Device}

This work uses the Realizer SLM 50 system, producing components out of a titanium alloy (Ti6Al4V). The process offers $25 \mu \mathrm{m}$ layer resolutions with a minimum feature size of approximately $150 \mu \mathrm{m}$, though this may vary depending on the material powder properties. While this process melts the powder in a specified pattern, small amounts of neighbouring powder also loosely adhere to weld boundaries during the process. Leaving a finish with a varying surface roughness; with titanium powder these adhesions reach sizes of $65 \mu \mathrm{m}$. Excessive surface roughness may be detrimental to obtaining optimum coaxial flow. 
Due to the complex internal geometry of the coaxial nozzle, this component has been oriented in such a way to minimize the requirements of any internal support structure during the build process.

The assembled design, shown in Figure 4, shows the orientation of the designed extruders at the predefined $45^{\circ}$ angle, as well as the coaxial nozzle and frame. All of which were produced through AM techniques. In addition to this, some components in the assembly were externally sourced. The polyether ether ketone (PEEK) heat shield was sourced commercially and fabricated through traditional manufacturing methods. The other elements externally sourced are the thermistors and nickel chromium resistance wire used to provide a feedback loop to heat the coaxial nozzle to temperatures up to $275^{\circ} \mathrm{C}$ (not shown in Figure 4).

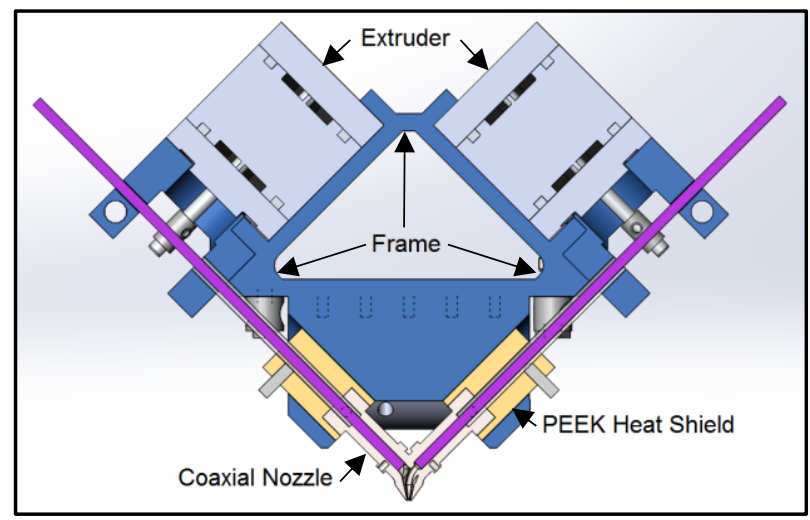

Figure 4 - CAD cross-section of assembled coaxial extrusion device

\section{Results AND Discussion}

\subsection{Measuring Coaxial Concentricity}

The concentricity of individually produced coaxial fibres is a key milestone for the successful completion of this work. Coaxial samples have been obtained by extruding two different colour PLA materials through the coaxial nozzle at velocities calculated previously. The tip was heated to $200^{\circ} \mathrm{C}$ and both flow rates controlled at $0.001(\mathrm{~m} / \mathrm{s})$ and $0.0002(\mathrm{~m} / \mathrm{s})$ for the external and internal material, respectively. The extruded distance was measured to be $10 \mathrm{~mm}$ of feedstock, with the resultant material free to flow without contact to the substrate. This allows the fibre to maintain its coaxial and cylindrical shape for ease of cross-sectional analysis. This procedure was conducted three times and the fibre was cross-sectioned by scalpel. The dissected fibre was examined using a Leica M205A microscope; a representation of a resultant coaxial fibre can be seen in Figure 5.

To assess the concentricity of the fibres, a method has been devised on the basis of the coaxial extrusion study by Cornock et.al. [17]. While the research does not indicate the exact process for assessing concentricity, it states that the desired measurement is between the longitudinal axis for both the internal and external materials. Therefore, the central axis for each material must be located and the distance between them measured. This is to be expressed as a percentage of the external material's radius to account for any dimensional variation between samples. The core material offset has been calculated using equation 1 , which is obtained with reference to Figure 5. 

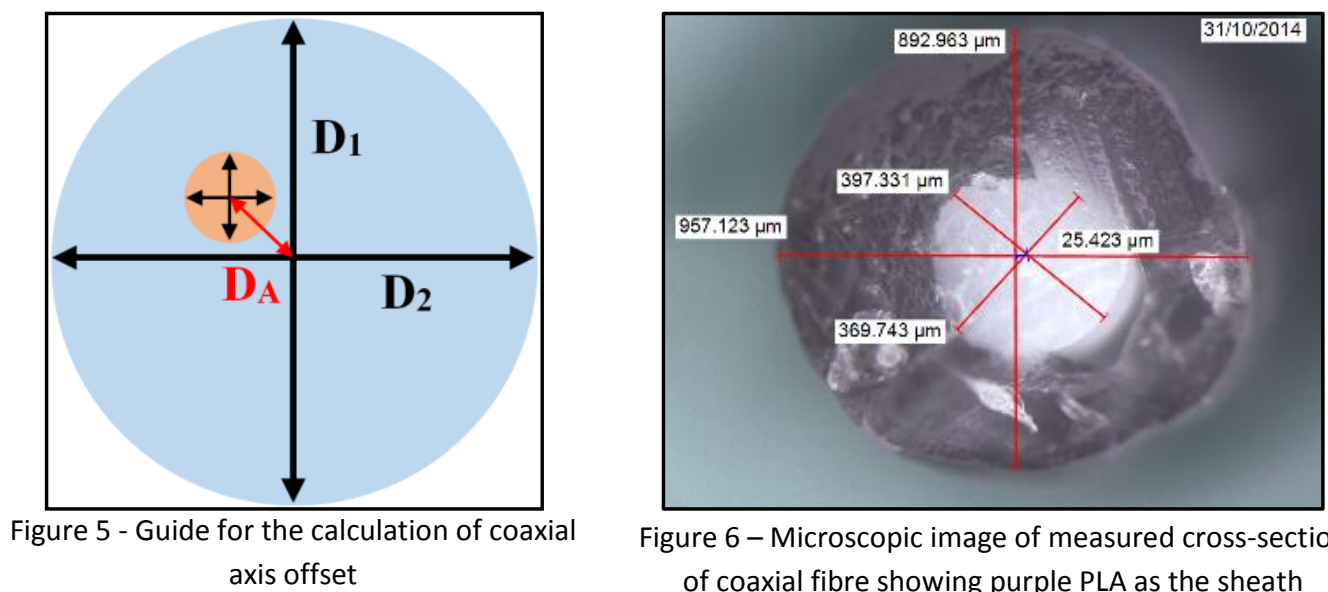

Figure 6 - Microscopic image of measured cross-section of coaxial fibre showing purple PLA as the sheath material and white PLA as the core material

$$
O_{A}=\frac{2 D_{A}}{\frac{D_{1}+D_{2}}{2}} \times 100
$$

where

$$
\begin{aligned}
& O_{A}=\text { Axis Offset }(\%) . \\
& D_{1}=\text { Diameter } 1 \text { of External Material. } \\
& D_{2}=\text { Diameter } 2 \text { of External Material. } \\
& D_{A}=\text { Distance from External Axis to Internal Axis. } \\
& \text { Note: } D_{1} \text { and } D_{2} \text { are to be perpendicular measurements about their midpoint }
\end{aligned}
$$

Figure 6 shows an analysed cross-section of a sample. By using this method to calculate offset, the effect of variation in the external diameter is minimized. By taking the average of both the vertical and horizontal diameters, it is possible to obtain a more relevant measure of offset. Failing to do so would result in skewed data as the resultant coaxial fibres may not be perfectly symmetrical in shape. Therefore, measuring an offset with respect to a non-symmetric shape would give ambiguous results.

\subsection{Effect of Flow-Rate on Concentricity}

With a means of assessing coaxial concentricity defined, the system can be tested to optimize parameters governing its performance. This section examines the impact of the rate at which feedstock is to be supplied to the hot-end (flow-rate) on coaxial concentricity. It is expected that an optimum flow rate will exist, where flow parameters are balanced, producing uniform concentric structures. Additionally, it is anticipated that lower flow rates will have less impact on die swelling. This is due to the molten material being held at pressure and temperature for longer, allowing the polymer chains to relax or detangle.

Figure 7 shows the effect of the flow-rate on coaxial concentricity, clearly indicating an optimum parameter range. Three cross-sectional measurements of fibres at flow-rates of 40 and $50 \mathrm{~mm} / \mathrm{min}$ produce average coaxial offsets of $2.89 \%$ and $3.5 \%$, respectively. This demonstrates the range over which the system will produce the most coaxial structures. This result was anticipated, while many mechanisms may contribute to these findings, the most significant influence is likely to be attributed to polymer melting time. 


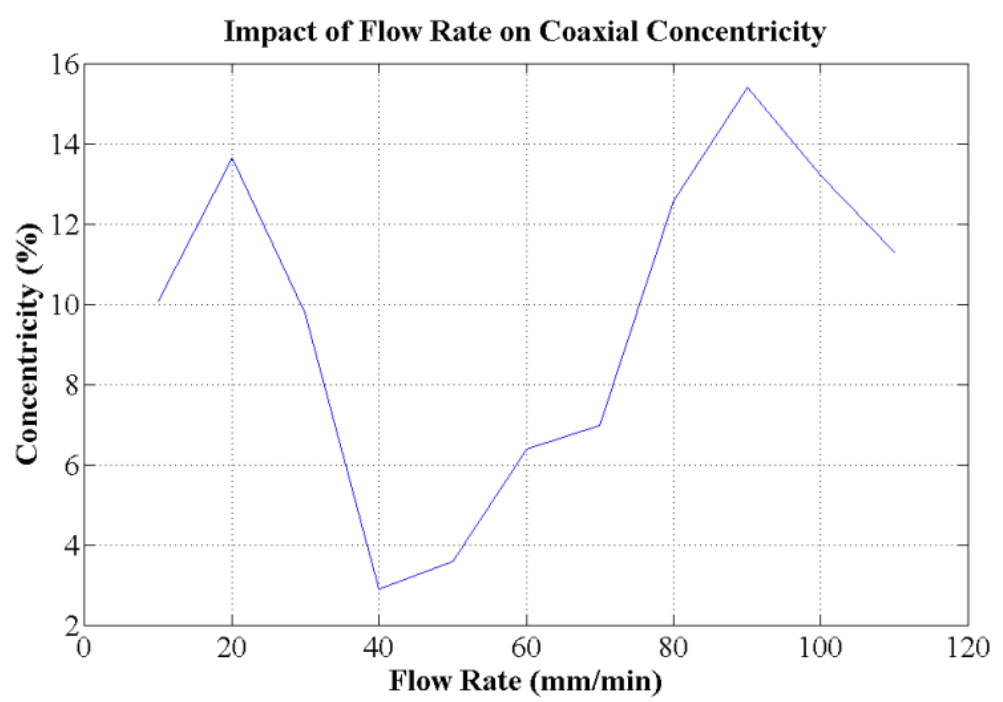

Figure 7 - Effect of flow rate on coaxial concentricity

While it was expected that varying the flow-rate would alter the fluid flow characteristics, the most influential factor was deemed to be time the feedstock spent in the heat chamber. By slowing the flowrate, the feedstock remains within the heat chamber for a longer duration, allowing it to reach higher temperatures. This in turn lowers the viscosity of the molten polymer, allowing it to flow more freely. Consequently, allowing the external material to more readily envelope the gap which exists between the two materials due to the internal nozzle wall.

This indicates two key outcomes, that flow-rate does impact coaxial concentricity, and also that concentricity is highly linked to material viscosity, and therefore print temperature. From this we can hypothesise that varying each materials heat chamber may positively impact the concentricity of the produced fibres.

Though the increased fluid temperature may produce more uniform coaxial structures, it may have detrimental effects on structures printed onto a substrate instead of freely extruded. The rate of cooling and material deformation resulting from depositing a higher temperature liquid will likely impact the coaxial quality of printed structures. Suggesting that printing at lower flow-rates may require external cooling on the resultant fibres to maintain their desired geometrical states.

Consequently, the increased temperature, and potential external cooling, is likely to induce undesirable internal stresses in the produced coaxial fibre as well as locking the extrudate in its polymer entangled state. The introduction of a sudden thermal gradient will cause a variation in cooling between the internal and external materials, causing stresses to form within the structure, altering its mechanical strength. However, it is likely the internal material will be shielded from this effect and will remain largely unaffected, implying a weaker sheath with a stronger core material.

\subsection{Explaining Discrepancies in Concentricity - Thermal Expansion}

As the aim of this work is to produce a concentric coaxial print material to the highest reliable resolution, we must consider the effect which thermal expansion plays on the extruded fibre. Any material to be printed in this nozzle will undergo a heating process for printing and a cooling process post-printing. As such, we must consider the temperature gradient between the molten material and the substrate or environment. Each material has its own unique print temperature as well as its own thermal expansion characteristics. 
The typical scenario of heat causes expansion and cold causes contraction plays an unusual role in the case of FDM systems. Typically, the heated material would be expected to decrease in size as it cools, and this will still be the case. The additional factor is the nature of the material being produced and the means of doing so. As the process involves a polymer undergoing temperature and pressure change through an extrusion die, it is expected that die swelling will influence the volume of the material immediately post-printing. The key factor is to consider is the ratio of thermal expansion against die swelling.

Taking PLA as the test material, the thermal expansion formula for the area is:

$$
\frac{\Delta \text { Area }}{\text { Area }_{o}}=\alpha \times \Delta T
$$

where

$$
\begin{aligned}
& \Delta \text { Area }=\text { Change in area }\left(\mathrm{mm}^{2}\right) \\
& \text { Area }_{o}=\text { Initial area }\left(\mathrm{mm}^{2}\right) \\
& \alpha=\text { Thermal coef ficient of expansion of PLA. } 7.4 e^{-4}\left({ }^{o} \mathrm{C}^{-1}\right) \\
& \Delta T=\text { Change in temperature. }\left({ }^{\circ} \mathrm{C}\right)
\end{aligned}
$$

Assuming an initial temperature of $200^{\circ} \mathrm{C}$, a final temperature of $25^{\circ} \mathrm{C}, \mathrm{Eq} .2$ is applied to both the internal and external nozzle material outputs. This results in the output diameters decreasing by $26.8 \mu \mathrm{m}$ and $59.9 \mu \mathrm{m}$ for the internal and external materials, respectively. Comparing these values to the dimensions found in a typical coaxial extrusion cross-section (Figure 6) appears to produce contradictory results. However, as the cross-section dimensions are similar to the coaxial nozzle output dimensions, the die swelling effect may be hiding these changes in area. This implies that the swelling factor is significantly influential on coaxial extrusion with research into die-swelling within FDM systems identifying a 1.051.3 swell ratio dependent upon the material used for printing[23].

This thermal expansion calculation has been used as a guide and will likely vary in practical application for the coaxial nozzle design. As the molten plastic passes from the heat chamber towards the tip, the material is slowly being cooled. The titanium alloy used to fabricate the coaxial nozzle exhibits adequate thermal conduction properties, though it is still anticipated that levels of heat loss are present as the distance from the heat chamber increases. This results in a lower change in temperature for the thermal expansion calculations, implying that the calculated values would be for extreme conditions.

\section{CONCLUSIONS AND Future Work}

This work has aimed to enhance the variability of structures which can be produced through means of FDM. To do so, a coaxial FDM system was designed and constructed, offering a wide range of control over the process. Three key goals have been realised to measure the success of the work produced. Firstly, we have produced a coaxial extrusion tip capable of accepting standard thermoplastic filament while having output dimensions of $1200 \mu \mathrm{m}$ and $400 \mu \mathrm{m}$ for the external and internal materials, respectively. Secondly, we have enhanced the reliability of the coaxial tip produced by optimizing its flow parameters and obtaining average coaxial offsets of $2.89 \%$, much lower than the literature value of $15 \%$. Finally, this work has reached a peak operating temperature of $275^{\circ} \mathrm{C}$ to maximize the range of materials to be used in the system. 
System and Process Development for Coaxial Extrusion in Fused Deposition Modelling

With these goals completed, this work contributes to research in additive manufacturing by:

- Developing a test platform for further experiments looking to fabricate with composite materials.

- Varying strength of printed parts or incorporating conductive fibres into the core-shell configuration.

- Providing a means to fabricate core-shell structures with high levels of coaxial concentricity, lending the nozzle design to the realm of bio-fabrication to produce high accuracy, complex, bio-scaffolds.

Subsequent research into the realm of coaxial FDM should look at producing smaller external diameter materials as well as altering the structure to allow for a three dimensional printing coating system. This process would involve supplying a solid, flexible material through the internal chamber and coating it with the external material. The flexibility of the solid material would allow it to be printed as if it were liquid, further enhancing the capabilities of coaxial structures as well as FDM.

\section{Acknowledgements}

The authors would like to acknowledge the Australian National Fabrication Facility (ANFF) for use of equipment and materials, Dr. Tony Romeo at the University of Wollongong Electron Microscopy Centre for his assistance in analysing structures and Mr. Fletcher Thompson for his technical contributions.

\section{References}

1. International, A., Standard Terminology for Additive Manufacturing Technologies. 2012, www.astm.org: West Conshohocken, PA.

2. $\quad$ Kruth, J.P., M.C. Leu, and T. Nakagawa, Progress in Additive Manufacturing and Rapid Prototyping. CIRP Annals - Manufacturing Technology, 1998. 47(2): p. 525-540.

3. Williams, J.C. and E.A. Starke Jr, Progress in structural materials for aerospace systems. Acta Materialia, 2003. 51(19): p. 5775-5799.

4. Petrovic, V., et al., Additive layered manufacturing: sectors of industrial application shown through case studies. International Journal of Production Research, 2010. 49(4): p. 1061-1079.

5. Song, Y., et al., Manufacture of the die of an automobile deck part based on rapid prototyping and rapid tooling technology. Journal of Materials Processing Technology, 2002. 120(1-3): p. 237-242.

6. Al Mortadi, N., et al., Design and fabrication of a sleep apnea device using computer-aided design/additive manufacture technologies. Proceedings of the Institution of Mechanical Engineers, Part H: Journal of Engineering in Medicine, 2013. 227(4): p. 350-355.

7. Hengsbach, S. and A. Lantada, Rapid prototyping of multi-scale biomedical microdevices by combining additive manufacturing technologies. Biomedical Microdevices, 2014. 16(4): p. 617627.

8. Murr, L.E., et al., Next-generation biomedical implants using additive manufacturing of complex, cellular and functional mesh arrays. Philosophical Transactions of the Royal Society A: Mathematical, Physical and Engineering Sciences, 2010. 368(1917): p. 1999-2032.

9. Ding, Y., et al., An integrated manufacturing system for rapid tooling based on rapid prototyping. Robotics and Computer-Integrated Manufacturing, 2004. 20(4): p. 281-288.

10. Harrysson, O.L.A., et al., Direct metal fabrication of titanium implants with tailored materials and mechanical properties using electron beam melting technology. Materials Science and Engineering C: Biomimetic and Supramolecular Systems, 2008. 28(3): p. 366-373. 
System and Process Development for Coaxial Extrusion in Fused Deposition Modelling

11. Leong, K.F., et al., Characterization of a poly-epsilon-caprolactone polymeric drug delivery device built by selective laser sintering. Bio-Medical Materials And Engineering, 2007. 17(3): p. 147-157.

12. Schmidt, M., D. Pohle, and T. Rechtenwald, Selective Laser Sintering of PEEK. CIRP Annals Manufacturing Technology, 2007. 56(1): p. 205-208.

13. Ramanath, H.S., et al., Melt flow behaviour of poly-e-caprolactone in fused deposition modelling. Journal of Materials Science: Materials in Medicine, 2008. 19(7): p. 2541-2550.

14. Design and Production of Wind Tunnel Testing Models with Selective Laser Sintering Technology Using Glass-Reinforced Nylon. Materials Science Forum, 2007. 532-533: p. 653-656.

15. Mortimer, M., B. Horan, and A. Stojcevski, Design for Manufacture of a Low-Cost Haptic Degree-Of-Freedom. International journal of electronics and electrical engineering, 2014. 2(2): p. 85-89.

16. Kim, G., et al., Coaxial structured collagen-alginate scaffolds: fabrication, physical properties, and biomedical application for skin tissue regeneration. Journal of Materials Chemistry, 2011. 21(17): p. 6165-6172.

17. Cornock, R., S. Beirne, and G.G. Wallace. Development of a Coaxial Melt Extrusion Printing process for specialised composite bioscaffold fabrication. in Advanced Intelligent Mechatronics (AIM), 2013 IEEE/ASME International Conference on. 2013.

18. matbase.com. General Purpose ABS. 2014 [cited 2014 4/11]; Available from: http://www.matbase.com/material-categories/natural-and-synthetic-polymers/commoditypolymers/material-properties-of-acrylonitrile-butadiene-styrene-general-purpose-gpabs.html\#properties.

19. Sin, L.T., A.R. Rahmat, and W.A.W.A. Rahman, 3 - Thermal Properties of Poly(lactic Acid), in Polylactic Acid, L.T.S.R.R.A.W.A. Rahman, Editor. 2013, William Andrew Publishing: Oxford. p. 109-141.

20. Sin, L.T., A.R. Rahmat, and W.A.W.A. Rahman, 4 - Chemical Properties of Poly(lactic Acid), in Polylactic Acid, L.T.S.R.R.A.W.A. Rahman, Editor. 2013, William Andrew Publishing: Oxford. p. 143-176.

21. Sin, L.T., A.R. Rahmat, and W.A.W.A. Rahman, 5 - Mechanical Properties of Poly(lactic Acid), in Polylactic Acid, L.T.S.R.R.A.W.A. Rahman, Editor. 2013, William Andrew Publishing: Oxford. p. 177-219.

22. Matweb.com. [cited 2014 4/11]; Available from: http://asm.matweb.com/search/SpecificMaterial.asp?bassnum=MTP641.

23. N. Turner, B., R. Strong, and S. A. Gold, A review of melt extrusion additive manufacturing processes: I. Process design and modeling. Rapid Prototyping Journal, 2014. 20(3): p. 192-204. 